\title{
Bioinformatic Analysis of Strawberry NDH-H Gene
}

\author{
Xiran Wang, Jiangang He, Haoru Tang \\ Sichuan Agriculture University, Chengdu, China.
}

\begin{abstract}
Keywords: $\mathrm{NDH}-\mathrm{H}$; research object; physical and chemical properties.
\end{abstract}
\begin{abstract}
NDH-H has always been known as a key factor of Hydrogen ion metabolism pathway in plant testa. Recent studies show that chlororespiration and cyclic electron flow mediated by NDH may coordinate to alleviate the over-reduction of stroma, thus to keep operation of CO2 assimilation at certain extent under heat stress condition. In this research we chosen NDH-H gene of 13 kinds species, downloaded their nucleotide and protein sequence from NCBI as the research object, found strawberry NDH-H gene via bioinformation analyses, constructed phylogenetic tree. At the same time, we analyzed the strawberry NDH-H gene of physical and chemical properties and its protein structure and so on. The phylogenetic tree showed that strawberry and Malus $\mathrm{x}$ domestica were closest relative. By the protein prediction, we found that the protein owed one proper signal peptide without obvious transmembrane regions.
\end{abstract}

\section{Introduction}

NDH-H has always been known as a key factor of Hydrogen ion metabolism pathway in plant testa. Recent studies show that chlororespiration and cyclic electron flow mediated by NDH may coordinate to alleviate the over-reduction of stroma, thus to keep operation of $\mathrm{CO} 2$ assimilation at certain extent under heat stress condition[1]. As the endosymbiont evolved into an organelle, the plastid retained a greatly reduced genome with many of the cyanobacterial genes being transferred to the host nuclear genome. Although most functional cp-tonuclear gene transfers occurred early in plastid evolution[2], such transfers have been a continual evolutionary process[3] , and the cp genome (plastome) of photosynthetic land plants typically encodes 68-82 conserved protein-coding genes[4-7]. The study chosen $\mathrm{NDH}-\mathrm{H}$-like gene of 13 kinds species, downloaded their nucleotide and protein sequence from NCBI as the research object, found strawberry NDH-H gene via bioinformation analyze so as to provide reference for further study and utilization.

\section{Result and Analysis}

\subsection{Strawberry NDH-H Find and Phylogenetic Tree Construction of NDH-H}

Run BLAST in the Fragaria ×ananassa database of GDR and Strawberry GARDEN respectively using Arabidopsis thaliana NDH-H as a sample. Select the sequence with ideal S and $\mathrm{C}$ value, we find that FAN_iscf00079132.1 is strawberry NDH-H.

We have chosen NDH-H-like gene of 13 kinds species (Table 1), downloaded their nucleotide and protein sequence from NCBI as the research object, analyzed

phylogenetic tree on the genetic distance base (Figure 1). The relationship between strawberry and petunia is closer than others, due to they all contain a lot of anthocyanins probably. 
Table 1. Basic information of ndh-h gene in different species

\begin{tabular}{|cccc|}
\hline Species & mRNA Accession Number & Protein Accession Number & Amino Acid Number \\
\hline Fragaria vesca & XM_004300664.2 & NP_764579841 & 2378 \\
Prunus persica & XM_007209819.2 & NP_1162557396 & 2,039 \\
Prunus avium & XM_021954618.1 & 1220048676 & 1,950 \\
Prunus mume & XM_008240407.2 & 1027110558 & 1,906 \\
Ziziphus jujuba & XM_016035329.1 & 1009146309 & 1,726 \\
Manihot esculenta & XM_021769310. & 1216280848 & 1,716 \\
Vitis vinifera & XM_010654642.2 & 1105496133 & 2,168 \\
Jatropha curcas & XM_012223503.2 & 1173833864 & 1,882 \\
Glycine max & XM_003525090.3 & 955321540 & 1,962 \\
Momordica charantia & XM_022293720.1 & 1229791775 & 1,870 \\
Cicer arietinum & XM_004503838.2 & 828317514 & 2,000 \\
Lupinus angustifolius & XM_019603041.1 & 1117349479 & 1,975 \\
Malus $x$ domestica & XM_008371310.2 & 1039874863 & 1,876 \\
\hline
\end{tabular}

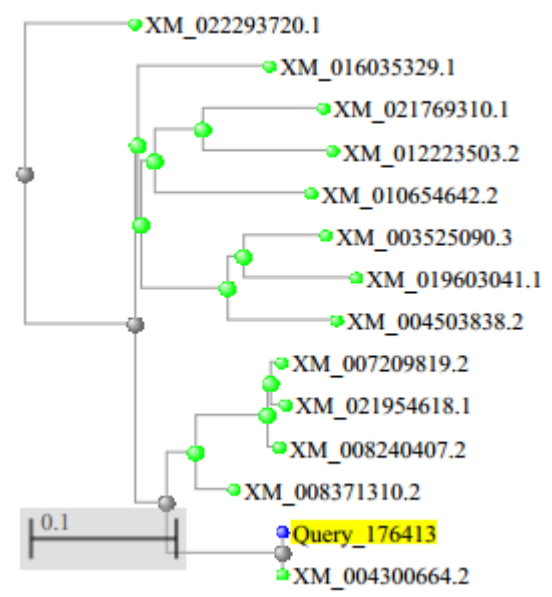

Figure 1. Phylogenetic Tree of Strawberry NDH-H.

\subsection{Strawberry Ndh-H Protein Physical Analysis}

Analyze strawberry NDH-H protein physical feature using ProtPram. Result shows as following: the protein is composed by $1611 \mathrm{bp}$, molecular weight is 132904.25 , theoretical pI is 5.00, electric neutrality, molecular formula is C4866H8163N1611O2075S319. Because the N-terminal of the sequence considered is A (Ala), the estimated half-life is 4.4 hours in mammalian reticulocytes (in vitro), 20 hours in yeast (in vitro) and 10 hours in Escherichia coli (in vitro). The instability index (II) is computed to be 45.68 , so the protein is unstable. The aliphatic index of the protein is 26.01 . Grand average of hydropathicity (GRAVY): 0.660.

Amino acid composition result is settings as following (Table 2): the peptide chain consists many Ala as $29.0 \%$.

Table 2. Strawberry ndh-h gene amino acid composition

\begin{tabular}{|ccc|}
\hline Amino Acid & Count & Percentage \\
\hline Ala (A) & 419 & $29.0 \%$ \\
Cys (C) & 319 & $19.8 \%$ \\
Gly (G) & 410 & $25.5 \%$ \\
Thr (T) & 463 & $28.7 \%$ \\
\hline
\end{tabular}

\subsection{Strawberry Ndh-H Protein Signal Peptide Forecast}

Forecast strawberry NDH-H protein signal peptide using SignalP 4.1. Result shows as following (Figure 2). There is one signal peptide in the NDH-H between 22th base and 23th base. 


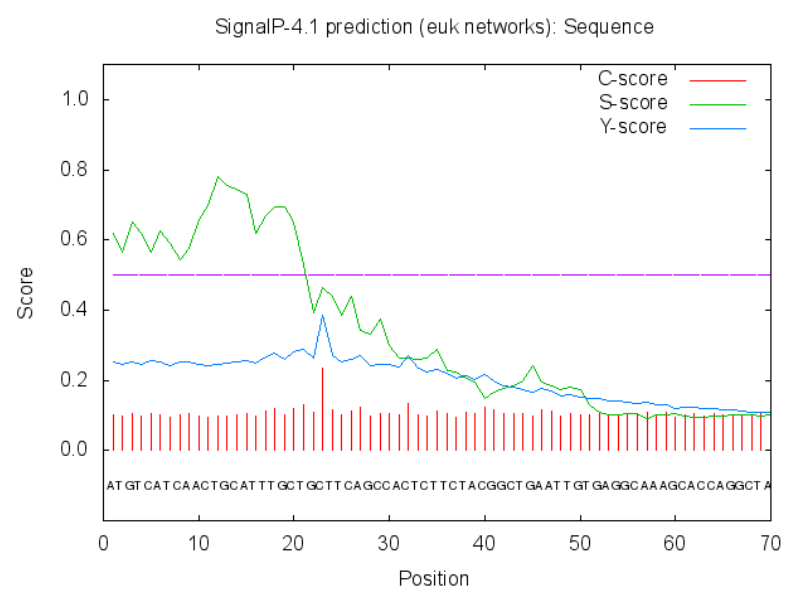

Figure 2. Signal peptide forecast of strawberry NDH-H.

\subsection{Strawberry Ndh-H Protein Transmembrane Domain Forecast}

Forecast strawberry NDH-H protein transmembrane domain using TMHMM Server. Result shows as following (Figure 3). There isn't transmembrane domain in the strawberry NDH-H, thus the protein is not cytomembrane receptor or located on cytomembrane.

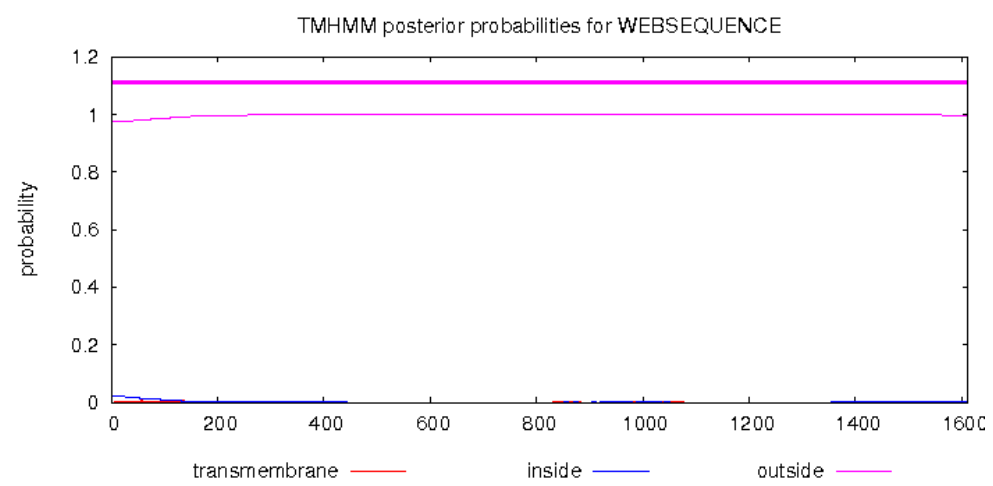

Figure 3. Transmembrane domain forecast of strawberry NDH-H.

\section{Materials and Methods}

Sequences access from NCBI (https://www.ncbi.nlm.nih.gov/). BLAST run in GDR (https://www.rosaceae.org/), and Strawberry GARDEN(http://strawberry-garden.kazusa.or.jp/). Phylogenetic tree is constructed via MEGA6.0, forecast strawberry NDH-H protein signal peptide using SignalP 4.1 (http://www.cbs.dtu.dk/services/SignalP/), forecast strawberry NDH-H protein transmembrane domain using TMHMM Server (http://www.cbs.dtu.dk/services/TMHMM/).

\section{Conclusion}

As suggested by Krause (2012) [8], parasitism may prompt adaptive gene loss. Some orchids still contain the whole set of NDH-related genes. The emergence of the heterotrophic stage of these orchids may have occurred very recently, and thus the NDH-related genes are still retained due to insufficient time for selection to eliminate them. Our analyses suggest that in contrast to nonorchid monocots, NDH complex genes in Orchidaceae may be evolving under relaxed selective constraints and may ultimately be lost. Further investigations into the correlation between $\mathrm{CO} 2$ fixation, growth habit, type of trophic strategy and NDH activity would be helpful to elucidate the mechanism and function of the NDH complex in plants. In addition to some photoautotrophic orchids, many non-orchid photoautotrophic plants also exhibit The exploration on strawberry TT12, which is forward gene to strawberry NDH-H, have been attempt in our lab, we would focus on the interaction between strawberry TT12 and NDH-H so as to provide reference for further study and utilization. 


\section{Acknowledgments}

I thank my teachers: Haoru Tang, for the directions, Jiangang He for the discussion. This work was financially supported by National Innovation Experiment Program for University Students (201510626005) fund.

\section{References}

[1]. Li Q, Yao Z J, Mi H. Alleviation of Photoinhibition by Co-ordination of Chlororespiration and Cyclic Electron Flow Mediated by NDH under Heat Stressed Condition in Tobacco:[J]. Front Plant Sci, 2016, 7(245):285-290.

[2]. Lin C, Chen J J W, Chiu C, et al. Concomitant loss of NDH complex - related genes within chloroplast and nuclear genomes in some orchids[J]. Plant Journal for Cell \& Molecular Biology, 2017, 90(5):994-998.

[3]. Timmis JN, Ayliffe MA, Huang CY, et al. Endosymbiotic gene transfer: organelle genomes forge eukaryotic chromosomes.[J]. Nat Rev Genet. 2004, 5(2):123-135.

[4]. Martin W, Herrmann R G. Gene transfer from organelles to the nucleus: how much, what happens, and Why?[J]. Plant Physiology, 1998, 118(1):9-17.

[5]. Simpson C L, Stern D B. The Treasure Trove of Algal Chloroplast Genomes. Surprises in Architecture and Gene Content, and Their Functional Implications[J]. Plant Physiology, 2002, 129(3):957-66.

[6]. Barrett C F, Freudenstein J V, Li J, et al. Investigating the path of plastid genome degradation in an early-transitional clade of heterotrophic orchids, and implications for heterotrophic angiosperms[J]. Molecular Biology \& Evolution, 2014, 31(12):3095.

[7]. Tiller N, Bock R. The Translational Apparatus of Plastids and Its Role in Plant Development[J]. Molecular plants, 2014, 7(7):1105-1120.

[8]. Krause K. Plastid Genomes of Parasitic Plants: A Trail of Reductions and Losses[M]// Organelle Genetics. Springer Berlin Heidelberg, 2012:79-103. 